\title{
Peripheral blood biomarkers in systemic sclerosis-associated interstitial lung diseases
}

\begin{abstract}
Interstitial lung disease (ILD) can lead to significant morbidity and excess mortality in systemic sclerosis (SSc) patients. The early identification of patients at risk of progressive ILD is the main challenges for treating this highly variable disease. Although high resolution CT (HRCT), lung function tests (PFTs), bronchial lavage fluid (BALF), lung biopsy can be used to detect SSc-ILD, it is necessary to find reliable predictors for the disease progression. Peripheral blood biomarkers offer the advantages of being readily obtained, non-invasive, and serially monitored. New insights during the past several years have underscored the epithelial and endothelial cell injury with inflammatory and immune activation impacting of clinically significant SSc-associated ILD, but the pathogenesis of SSC-ILD is unknown. Some serum biomarkers including SP-D, KL-6, IL-9, MMPs, MCP-1, Defensins, IL-8 CXCL10 and CCL18 have identified to be associated with SSC-ILD. Future more cohorts, well-designed and long-term studies are needed to find more prospective biomarkers that influence SSc-ILD disease progression.
\end{abstract}

Keywords: systemic sclerosis (SSc) • interstitial lung disease (ILD) • peripheral blood biomarkers

\section{Introduction}

Systemic sclerosis (SSc) is a disorder of the connective tissue characterized by fibrosis of the skin, vascular abnormalities, and presence of auto-antibodies [1]. It is characterized by excessive deposition of extracellular matrix. Therefore, there is significant heterogeneity in organ progression and prognosis.

Interstitial lung disease (ILD) is a heterogeneous group of parenchymal lung disorders that share common radiologic, pathologic, and clinical manifestations [2]. It is characterized by lung parenchyma damage, accompanied by inflammation and fibrosis, and fibrosis is often incurable. The fibrosing forms of ILD are often incurable, and are associated with significant morbidity and mortality [2].

$\mathrm{SSc}$ is often accompanied by ILD. The incidence of SSc-ILD in the relevant literatures ranges from $45 \%$ to $90 \%[2,3]$. A recent European League Against Rheumatism Scleroderma Trials and Research analysis revealed in a cohort of 3,656 SSc patients that ILD was present in $53 \%$ of cases with diffuse cutaneous SSc and in 35\% of cases with limited cutaneous SSc [4]. Pulmonary involvement is the leading cause of death in SSc patients due to pulmonary fibrosis or pulmonary hypertension. By current data, 34\% of SSc deaths are due to SSc-ILD [5], while in developing countries the death rate is as high as $44.6 \%$.

ILD can be a subclinical form (imaging or physiological abnormalities, but not accompanied by symptoms), chronic progression, or may occur in an explosive, life-threatening manner. The clinical course of ILD is divided into two subtypes: acute/ sub-acute interstitial pneumonia (A/SIP), also called rapidly progressive ILD (RP-ILD), and chronic IP (CIP). The pathophysiological classification includes usual interstitial pneumonia (UIP), nonspecific interstitial pneumonia (NSIP), organizing pneumonia (OP), diffuse alveolar damage (DAD), desquamative interstitial pneumonia, and lymphoid interstitial pneumonia [6].

NSIP is the more common subtype of inflammation in ILD. $77 \%$ of SSc-ILD are NSIP [7]. A large number of clinical and pathological studies have confirmed that high resolution CT (HRCT) pattern in patients are correlated with pathologic NSIP

\author{
Ciyuan Yu, Shuli Song \& Juan Chen* \\ Rheumatology Department, Xiamen \\ University and Fujian Medical University of \\ Affiliated First Hospital, Xiamen, China \\ *Author for correspondence: \\ juchen@xmu.edu.cn
}


and pathologic UIP $[8,9]$. NSIP pattern is associated with better patient outcome than UIP pattern. It includes reticular, frosted glass shadows, hollow, thickened honeycomb lung nodules, emphysema, bronchial vascular bundles, bullae, traction bronchiectasis, cobblestone like appearance, bronchial tree, bronchiectasis and so on [10]. The most common manifestation of NSIP is lobular reticular formation in the pleural and basal regions [11,12]. UIP is mainly represented by grid or honeycomb shadow. Different patterns in HRCT can reflect NSIP and UIP [6]. The extent of ILD lesions can be graded according to HRCT [13]. At present, the commonly used methods for clinical detection of ILD are HRCT, pulmonary function tests (PFTs), bronchoalveolar lavage fluid (BALF), lung biopsy. HRCT has now become the most common and sensitive imaging method for diagnosing ILD as it offers the most detailed images of the lungs [14-16]. HRCT and PFTs are the most sensitive to change [17]. The specificity of PFTs is low [18]. Bronchoalveolar lavage fluid is a sensitive marker for the future development of SSc in patients with ILD, but it is invasive test. Although the specificity of lung biopsy is high, the clinical operation is difficult and the practicability is low. Therefore, the search for both economical and non-invasive methods of examination is particularly important. Biomarkers of serum are not only easy to obtain, noninvasive, but also can be monitored continuously [19], moreover, the early diagnosis of subclinical ILD in SSc is an effective and feasible way to identify SScILD, which can improve the prognosis and mortality.

Though it is not clear about the pathogenesis of systemic sclerosis associated with interstitial lung diseases, serum biomarkers, genes and lifestyle are involved in SSc-ILD progression [20]. Unknown factors initiate immune dysfunction between epithelial and vascular endothelial cell, activate fibroblast function and extracellular matrix production. The combination of epithelial and endothelial cell injury as well as inflammatory and immune activation progress pulmonary fibrosis in SSc [19].

This paper focuses on peripheral blood biomarkers in SSc-ILD and other connective tissue disease (CTD) associated ILD.

\section{$\mathrm{KL}-6$}

Krebs von den Lungen-6 (KL-6) is an important serum marker for ILD [21,22]. It is a high molecular weight, mucin like glycoprotein secreted by type-II alveolar pneumocytes and bronchial epithelial cells in response to cellular damage and regeneration in patients with ILD [21]. KL-6 is a mucin-associated glycoprotein, which may be a trigger for TGF- $\beta$ signaling and fibrosis [23].

The level of KL-6 as a predictive factor could be used to identify the clinical development of ILD [24]. Hideaki et al. [25] retrospectively analyzed the medical records of 29 patients with SSc-ILD. Measurement of serum KL-6 levels, pulmonary function tests, and HRCT performed in parallel were reviewed. They found serum KL-6 correlated positively with diffusing capacity of the lung for carbon monoxide (DLCO)(\% predicted) and disease extent on HRCT, and the changes in serum levels of KL-6 were significantly related to the changes in forced vital capacity (FVC) in SSc -associated ILD. Their study suggests KL-6 can be a useful monitoring tool of SSc-ILD activity.

Masataka et al. investigated the relationship between SSc-ILD and prognosis [26]. They chose fifty patients with early-stage SSc-ILD who had never received disease-modifying drugs and were either observed for $\geq 10$ years or died from ILD-related causes, and the Cox proportional hazard model was used to identify initial factors that correlated with end stage lung disease (ESLD) development. They found elevated KL-6 at initial assessment was highly correlated with ESLD development and the initial KL-6 level correlated with the forced vital capacity decline rate. Baseline serum KL-6 is a biomarker potentially useful for predicting FVC decline.

The KL-6 in the peripheral serum was described earlier, and the relationship between KL- 6 and the severity and the activity of SSc-ILD in bronchoalveolar lavage fluid was studied [27]. Fifteen patients with early SSc and 12 healthy controls were subjected to BALF. Serum KL-6 were increased in SSc patients and correlated with BALF concentration of eosinophils. Patients with more widespread ground glass opacities (GGO) on HRCT were characterized in BALF by a higher eosinophil count and in serum by higher KL-6. Therefore, KL-6 levels from BALF and serum being correlated to 
findings on HRCT suggest that KL-6 can be used as a useful biomarker of presence and extent of lung fibrosis in SSc patients.

KL-6 is also an important biomarker in other CTD-ILD. Higher levels of KL-6 was a diagnostic marker in 68 CTD-ILD group than in either 45 CTD without pulmonary involvement group or 45 healthy control group [28]. CTD-ILD included 12 Sjogren syndrome related interstitial lung disease (SS-ILD), 18 rheumatoid arthritis related interstitial lung disease (RA-ILD), 3 polymyositis/ dermatomyositis related interstitial lung disease (PM/DM-ILD). Interestingly, in other studies on PM/DM-ILD, the level of serum KL-6 was significantly higher [29-32]. KL-6 could be treated as a biomarker of disease activity and therapy efficacy of CTD-ILD.

\section{SP-D}

Surfactant, a lipoprotein complex, was originally described for its essential role in reducing surface tension at the air-liquid interface of the lung. However, it is now recognized as being a critical component in lung immune host defense. They include SP-B and SP-C and hydrophilic proteins SP-A and SP-D [33]. SP-D levels are more sensitive than SP-A in detecting ILD as defined by CT [19]. The sensitivities and specificities for detecting CT-positive ILD in 42 patients with SSc were $33 \%$ and $100 \%$ for SP-A and $77 \%$ and $83 \%$ for SP-D, respectively [34].

In a small but prospective study of 35 patients with SSc-ILD followed over 1-10 years, SP-D levels were seen to definitely increase over time in 9 out of the 10 patients with worsening ILD, as defined by changes in symptoms, lung function, and imaging, compared to mild increases in only 3 out of 25 patients with stable or improving SSc-ILD [35]. Therefore, SP-D is closely related to SSc-ILD.

A current study [25] retrospectively analyzed the medical records of 29 patients with SSc. Measurement of serum SP-D levels, pulmonary function tests, and HRCT performed in parallel were reviewed. Serum SP-D concentrations were also measured with commercially available ELISA kits. They considered a decline in FVC of $>0.08 \mathrm{~L} /$ year on the ROC curve and found a higher serum level of SP-D was a significant predictor of potential FVC decline in patients with SSc-ILD. Serum levels of SP-D correlate with SSc- ILD activity.
In addition, Takahashi $\mathrm{H}$ et al. [34] found a less-invasive and lung-specific clinical biomarker. They measured serum levels of SP-D by sandwich enzyme-linked immunosorbent assays in 42 patients with progressive systemic sclerosis (PSS) to evaluate their significance in relation to the presence of ILD and to assess their diagnostic merits. They found the levels of SP-D in sera were significantly higher in the CT-positive ILD group than in the CTnegative ILD group. The research shows imaging and laboratory tests complement each other and support each other, suggesting serum SP-D is a clinically useful tool for detecting ILD complicated with PSS.

Similar results were in other CTD-ILD. SP-D levels were significantly increased in 113 RA-ILD, and strengthened the presence of clinically evident and subclinical RA-ILD on computed tomography scan in two independent RA cohorts and may facilitate identification of RA-ILD at an earlier stage, potentially leading to decreased morbidity and mortality [36]. Another study [37] found that the serum SP-D level in PM/DM patients with ILD was significantly higher than in those without ILD. The incidences of decreased vital capacity (\%VC) and of decreased diffusing capacity of carbon monoxidase were also significantly greater in patients with an elevated SP-D level than in those with a normal level. SP-D was also significantly elevated in PM/DM patients with ILD compared with those in the patients without ILD [30].

IL-9

Interleukin 9 (IL-9) is a T cell-derived cytokine that was initially designated as a Th2 cytokine. Interleukin 9 targets cells of the lymphoid, myeloid, and mast cell lineages and likely contributes to the development of autoimmune diseases [38-41]. Yanaba $K$ et al. [42] determined serum IL-9 levels and their clinical associations in patients with SSc. Serum IL-9 levels were examined by ELISA in 71 patients with SSc and the Mann-Whitney U test was used to compare IL-9 levels. Serum IL-9 level was increased in patients with $\mathrm{SSc}$, and was associated with lower frequency and severity of pulmonary fibrosis in SSc. IL-9 could be a protective factor against the development of pulmonary fibrosis in this disease. They studied the relationship between the $\mathrm{SSc}$ and the clinic, but there was no convincing combination of imaging findings. 
Other study [43] investigated the expression of IL-9 levels in SSc-ILD patients and the association of IL-9 with disease activity to better understand the relationship and immunopathological role of IL-9 in SSc-ILD. The peripheral blood samples were obtained from 9 patients with SSc. Determination of ILD was according to the HRCT performance, and the patients were divided into a SSc-ILD group and a simple SSc group according to presence or absence of ILD. They found serum IL-9 levels were significant higher in SScILD and simple SSc patients than they were in healthy controls and that the levels were elevated in SSc-ILD patients compared with simple SSc patients. They also found the serum IL-9 levels were negatively correlated with $\mathrm{FVC} \%$ and DLCO\% in patients with SSc. Therefore, IL-9 levels were elevated not only in patients with simple SSc but also in those with SSc-ILD, demonstrating that IL-9 may contribute to the progression of interstitial lung injury in SSc and the IL-9 levels are gauges of the degree of damage to pulmonary function in patients with SSc-ILD. The sample size is too small to convince them of the experiment.

Serum IL-9 levels were also studied in other CTD-ILD. Serum IL-9 levels were significantly higher in 29 CTD-ILD and simple 32 CTD patients than 20 healthy controls. The IL-9 levels were elevated in CTD-ILD including 7 RA-ILD and 7 DMILD patients compared with simple CTD patients [43].

\section{MMPs}

The matrix metallo-proteinases (MMPs) are a family of zinc- and calcium-dependent endopeptidases that have the ability to degrade all major connective tissue matrices. The MMPs are involved in a variety of tissue remodeling under normal and pathological conditions. The MMPs can cleave most of the constituents of the ECM including collagen, proteoglycan, laminin, fibronectin and elastin [44]. The potent proteolytic activities of MMPs are mainly regulated by the balance with specific tissue inhibitors of Matrix metallo-proteinases (TIMP). Excessive or inappropriate expression of MMP may contribute to the pathogenesis of tissue destructive processes in a wide variety of diseases including lung diseases [45].

MMP-9, a type-IV collagenase, also called gelatinase B because of its ability to break down gelatin, has substrate specificity for a variety of
ECM constituents such as native collagens IV and $V$, gelatin, elastin and vitronectin and has been observed to play an important role in lung tissue remodelling in an experimental model of lung fibrosis [44]. MMP-9 is produced constitutively by neutrophils and eosinophils, stored in granules and immediately released in the pro-form upon chemokine stimulation [46].

One study [47] emphasized that MMP-9 plays an important role in ECM refactoring of SScILD by measuring levels of MMP-9 in BAL fluid from SSc patients with signs of ILD on HRCT, in SSc patients with normal HRCT and in healthy controls and related these levels to lung function. They chose nine SSc patients with ILD, seven SSc patients without ILD and 16 ages- and sex-matched healthy controls. Their experimental results were total MMP-9 and pro-MMP-9 levels were significantly increased in BAL fluid from the nine SSc patients with signs of ILD on HRCT compared to levels in healthy controls and compared to levels in SSc patients with normal HRCT. In SSc patients with ILD, calculated active MMP-9 levels were significantly higher than in SSc patients without ILD and tended to be higher than in healthy controls. And they proved the levels of total-MMP-9 and proMMP-9 were all negatively associated with TLC (total lung capacity), MMP-9 levels, regardless if expressed as total-, pro- or active MMP-9 were all inversely associated with TLC, consistent with a role for alveolar MMP9 in remodeling and lung volume reduction.

MMP-12 is a member of MMPs secreted extracellularly [48] and expressed in several cell types, and degraded several ECM proteins. Increasing evidence also indicates that MMP12 is involved in types of pathological lung tissue remodeling such as chronic pulmonary inflammation and fibrosis [49-51].

Serum MMP-12 levels were raised in SScILD compared with patients without ILD by ELISA and correlated with severity of lung restriction from $72 \mathrm{SSc}$ patients and 42 healthy controls. Immunohistochemical expression of MMP-12 were found in capillary vessels, inflammatory cells, alveolar macrophages and fibroblasts in the thickened alveolar septa in SSc-ILD, while faint expression in normal lung tissue, which were from lung biopsies of three SSc-ILD patients and five healthy controls. The conclusion is that MMP-12 levels are 
increased in SSc patients and involved in the pulmonary fibrosis of SSc-ILD [52].

Although there has been no literature on the relationship between MMP-7 and SSc-ILD, MMP-7 were identified by multiplex ELISA as potential biomarkers for RA-ILD in 133 Chinese RA cohort (50 RA-no ILD, 41 RAILD, 42 RA-indeterminate ILD) as well as in an independent cohort of US patients with RA and different stages of ILD (22 RA-no ILD, 49 RA-ILD, 15 RA-indeterminate ILD) [13]. Levels of MMP-7 were elevated in the serum of RA-ILD, supporting their value as pathogenically relevant biomarkers that can contribute to noninvasive detection of this extra-articular disease complication.

\section{CXCL-10}

CXC chemokines attract neutrophils and promote their adhesion to endothelial cells [53]. CXCL10 activate the function of Th1 lymphocytes secreted interferon (INF). CXCL10 levels are a reliable marker of aggressive Th1-mediated autoimmune disease [54]. The high serum levels of Th1 chemoattractant CXCL10 and Th2 chemical attractants were found in only one crosssectional study in SSc [55].

One study investigated serum levels of CXCL10 in ILD associated with SSc patients. CXCL10 level from patients with SSc was significantly higher than that from healthy volunteers, and the median CXCL10 level from SSc patients with ILD was significantly higher than that from SSc patients without ILD. CXCL10 is specifically increased in SSc patients with the lung involvement. CXCL10 plays a role in scleroderma lung disease. ILD were identified by CT in the study $[56,57]$. The untreated SSc patients with relatively short disease duration had high serum levels of CXCL10. Meanwhile high values of CXCL10 seemed to be associated with more severe clinical phenotype, namely with the presence of lung fibrosis. This provides a reference for the early diagnosis of SSc, especially SSc-ILD [57].

The similar results as MMP7 in the same study, serum CXCL-10 levels were elevated in RA-ILD comparing with RA no ILD [13].

\section{MCP-1(CCL-2)}

CCL2 is known as monocyte chemo-attractant protein-1 (MCP-1). MCP-1 is a member of the C-C chemokines [58]. In vivo studies suggest that MCP-1 recruits monocytes/ macrophages to sites of inflammation in a wide variety of pathological conditions, including ILD [59].

The plasma level of CCL2 is correlated with FVC value in SSc. However, there were no correlation between ILD severity and primary fibrotic genes such as collagen in the study [60]. That might be because skin fibrosis peaks early during the course of SSc and improves later [61], while fibrosis in pulmonary tissue continues to progress even at later stages of disease [62].

A study [63] examined serum levels, spontaneous production by peripheral blood mononuclear cells (PBMC), and histological distribution in the affected skin, of MCP-1. Elevated serum levels of MCP-1 significantly correlated with the presence of pulmonary fibrosis. MCP-1 was expressed in mononuclear cells or vascular endothelial cells in 41\% (9/22) of SSc patients. The frequency of infiltrating mononuclear cells and endothelial cells that produced MCP-1 was significantly higher in $\mathrm{SSc}$ patients with early onset than in $\mathrm{SSc}$ patients with late onset. The abnormal accumulation of macrophages in the lungs may result from augmented local production of $\mathrm{MCP}-1$ in the affected lungs of SSc, leading to elevated serum MCP-1 levels in patients with SSc. MCP-1 may play an important role in the development of pulmonary fibrosis in SSc.

Serum MCP-1 levels were significantly elevated in PM/DM patients with ILD compared with those in the patients without ILD in other study [30].

\section{CCL18}

The C-C motif chemokine ligand 18(CCL18) secreted from the alveolar macrophages can be activated by the Th 2 cytokines, and increased in ILD such as SSc-ILD. Higher rates of disease progression occurred in higher serum CCL18 levels in a longitudinally over a 4-yr observation in $\mathrm{SSc}$ patients for the risk complication events which were defined as a $10 \%$ decrease from baseline of total lung capacity or forced vital capacity \% predicted, or death, serum CCL18 levels. They found serum CCL18 levels are an accurate predictive biomarker for higher risk of subsequent scleroderma lung disease worsening [64].

Similarly, there is a study [65] assessed CCL18 
in supernatants of cultured BAL cells as well as BAL fluid and serum samples from 43 patients with IIPs, 12 patients with SSc, and 23 healthy control subjects. CCL18 production by BAL cells and serum CCL18 concentrations reflect pulmonary fibrotic activity in patients with IIPs and those with SSc. Monitoring changes in CCL18 production might be an extraordinarily useful tool in clinical practice and in studies aimed at evaluating new approaches for treatment of fibrotic lung diseases.

\section{Defensins and IL-8}

The early studies found IL-8 were correlated with the respective BAL cell differentiation, lung function, and thoracic HRCT score in SSc patients. IL-8 levels were significantly increased in the ILD-positive SSc patients than ILD-negative, and negative correlations between the predicted DLCO levels and the BALF concentrations of IL-8. Predicted FVC values also correlated negatively with the BALF and IL-8 levels. IL-8 is the mostpromising candidates for a targeted therapy in SSc-associated ILD [66].

Defensins are human neutrophil peptides (HNPs). It is a cationic antibacterial peptide rich in cysteine, which plays an important role in innate immunity and regulates the adaptive immunity of the host [67]. IL-8 is a potent neutrophil chemo-attractant [68]. Elevated plasma levels of HNPs were higher in acute exacerbation of interstitial pneumonia than in stable interstitial pneumonia, but not specific enough to serve as candidate biomarkers of acute exacerbation of interstitial pneumonia [66]. Recent study found BALF levels of HNPs and IL-8 were higher in SSc-ILD than in healthy controls, and are associated with various clinical disease parameters [68]. It is the first time to suggest that HNPs and IL-8 in BALF play a critical role in the pathogenesis of SSc-ILD [68]. HNPs induce the production of cytokines such as IL-8 and growth factors, which play pivotal roles in pulmonary fibrosis $[66,69]$. HNPs derived from neutrophils produce IL-8 in the lung. Defensins and IL-8 play a critical role in SSc-ILD [68]. This conclusion needs to be confirmed by increased patients number with no SSc-ILD controls and more cohorts with longitudinally study.

Kawasumi $\mathrm{H}$ [70] analyzed the associations between disease activity and levels of serum cytokines in PM/ DM. IL-8 were significantly correlated with rapidly progressive interstitial lung disease with $\mathrm{PM} / \mathrm{DM}$, especially in the case of the RP-ILD subset than in the nonILD subset or the chronic ILD subset.

\section{Conclusion}

Interstitial lung disease can lead to significant morbidity and excess mortality in systemic sclerosis patients. The early identification of patients at risk of progressive ILD is the main challenges for treating this highly variable disease. Although high resolution $\mathrm{CT}$, lung function tests, bronchial lavage fluid, lung biopsy can be used to detect SScILD, it is necessary to find reliable predictors for the disease progression. Peripheral blood biomarkers offer the advantages of being readily obtained, non-invasive, and serially monitored.

The epithelial and endothelial cell injury with inflammatory and immune activation impacting of clinically significant SScassociated ILD, but the pathogenesis of SScILD is unknown.

The improved biomarkers development in SScILD including SP-D, KL-6, IL-9, MMPs, MCP-1, Defensins Defensins, IL-8 CXCL10 and CCL18. CCL18, SP-D and KL-6 are more readily available biomarkers in clinic for SSc-ILD. Future more cohorts, well-designed and long-term studies are needed to find more prospective biomarkers that influence CTDILD disease progression.

\section{Disclosures}

The authors have no financial relationships or conflicts of interest to disclose.

\section{References}

1. Crincoli V, Fatone L, Fanelli M et al. Orofacial Manifestations and Temporomandibular Disorders of Systemic Scleroderma: An Observational Study. Int. J. Mol. Sci. 17(7) (2016).

2. Castelino FV, Varga J. Interstitial lung disease in connective tissue diseases evolving concepts of pathogenesis and management. Arthritis. Res. Ther. 12(4), 213 (2010).

3. Iudici M, Moroncini G, Cipriani P et al. Where are we going in the management of interstitial lung disease in patients with systemic sclerosis? Autoimmun. Rev. 14(7), 575-578 (2015).

4. Walker UA, Tyndall A, Czirjak L et al. Clinical risk assessment of organ manifestations in systemic sclerosis: a report from the EULAR Scleroderma Trials And Research group database. Ann. Rheum. Dis. 66(6), 754-763 (2007). 
5. Okamoto M, Fujimoto K, Sadohara J et al. A retrospective cohort study of outcome in systemic sclerosis-associated interstitial lung disease. Respir. Investig. 54(6), 445-453 (2016).

6. Society ATSER. American Thoracic Society European Respiratory Society International Multidisciplinary Consensus Classification of the Idiopathic Interstitial Pneumonias. Am. J. Respir. Crit. Care. Med. 165(2), 277-304 (2002).

7. Bouros D, Wells AU, Nicholson AG et al. Histopathologic subsets of fibrosing alveolitis in patients with systemic sclerosis and their relationship to outcome. Am. J. Respir. Crit. Care. Med. 165(12), 1581-1586 (2002).

8. Walsh SL, Wells AU, Sverzellati N et al. Relationship between fibroblastic foci profusion and high resolution $\mathrm{CT}$ morphology in fibrotic lung disease. BMC Med. 13, 241 (2015).

9. Mink SN, Maycher B. Comparative manifestations and diagnostic accuracy of high-resolution computed tomography in usual interstitial pneumonia and nonspecific interstitial pneumonia. Curr. Opin. Pulm. Med. 18(5), 530-534 (2012).

10. Tanaka N, Kim JS, Newell JD et al. Rheumatoid arthritis-related lung diseases CT findings. Radiology. 232(1), 81-91 (2004).

11. Hwang JH, Misumi S, Sahin $\mathrm{H}$ et al. Computed tomographic features of idiopathic fibrosing interstitial pneumonia comparison with pulmonary fibrosis related to collagen vascular disease. J. Comput. Assist. Tomogr. 33(3), 410-415 (2009).

12. DA L. Lung disease related to collagen vascular disease. J. Thorac. Imaging. 24(4), 299-309 (2009).

13. Chen J, Doyle TJ, Liu Y et al. Biomarkers of rheumatoid arthritis-associated interstitial lung disease. Arthritis. Rheumatol. 67(1), 28-38 (2015).

14. Flaherty KR, King TE Jr., Raghu G et al. Idiopathic interstitial pneumonia: what is the effect of a multidisciplinary approach to diagnosis? $\mathrm{Am}$. J. Respir. Crit. Care. Med. 170(8), 904-910 (2004).

15. Bussone G, Mouthon L. Interstitial lung disease in systemic sclerosis. Autoimmun. Rev. 10(5), 248-255 (2011).

16. Verschakelen JA. The role of high-resolution computed tomography in the work-up of interstitial lung disease. Curr. Opin. Pulm. Med. 16(5), 503-510 (2010).

17. Robinson TE, Leung AN, Northway WH et al. Spirometer-triggered high-resolution computed tomography and pulmonary function measurements during an acute exacerbation in patients with cystic fibrosis. J. Pediatr. 138(4), 553-559 (2001).

18. Schreiber BE, Valerio CJ, Keir GJ et al. Improving the detection of pulmonary hypertension in systemic sclerosis using pulmonary function tests. Artbritis. Rheum. 63(11), 3531-3539 (2011).

19. Lota HK, Renzoni EA. Circulating biomarkers of interstitial lung disease in systemic sclerosis. Int. J. Rheumatol. 2012, 21439 (2012).
20. Ludwicka-Bradley A, Silver RM, Bogatkevich GS Coagulation and autoimmunity in scleroderma interstitial lung disease. Semin. Arthritis. Rheum. 41(2), 212-222 (2011).

21. Hu Y, Wang LS, Jin YP et al. Serum Krebs von den Lungen-6 level as a diagnostic biomarker for interstitial lung disease in Chinese patients. Clin. Respir. J. 11(3), 337-345 (2017).

22. Ishikawa N, Hattori N, Yokoyama A et al. Utility of KL-6/MUC1 in the clinical management of interstitial lung diseases. Respir. Investig. 50(1), 3-13 (2012).

23. Ohshimo S, Yokoyama A, Hattori N et al. KL-6, a human MUC1 mucin, promotes proliferation and survival of lung fibroblasts. Biochem. Biophys. Res. Commun. 338(4), 1845-1852 (2005).

24. Ohnishi H, Yokoyama A, Kondo Ket al. Comparative study of KL-6, surfactant protein-A, surfactant protein-D, and monocyte chemoattractant protein-1 as serum markers for interstitial lung diseases. $\mathrm{Am}$. J. Respir. Crit. Care. Med. 165(3), 378-381 (2002).

25. Yamakawa $\mathrm{H}$, Hagiwara E, Kitamura $\mathrm{H}$ et al. Serum KL-6 and surfactant protein-D as monitoring and predictive markers of interstitial lung disease in patients with systemic sclerosis and mixed connective tissue disease. J. Thorac. Dis. 9(2), 362-371 (2017).

26. Kuwana M, Shirai Y, Takeuchi T. Elevated Serum Krebs von den Lungen-6 in Early Di. J. Rheumatol. 43(10), 1825-1831 (2016).

27. Hesselstrand R, Wildt M, Bozovic G et al. Biomarkers from bronchoalveolar lavage fluid in systemic sclerosis patients with interstitial lung disease relate to severity of lung fibrosis. Respir. Med. 107(7), 1079-1086 (2013).

28. Oguz EO, Kucuksahin O, Turgay $\mathrm{M}$ et al. Association of serum KL-6 levels with interstitial lung disease in patients with connective tissue disease: a cross-sectional study. Clin. Rheumatol. 35(3), 663666 (2016).

29. Fathi M, Barbasso Helmers S, Lundberg IE. KL-6: a serological biomarker for interstitial lung disease in patients with polymyositis and dermatomyositis. $J$. Intern. Med. 271(6), 589-597 (2012).

30. Chen F, Lu X, Shu X et al. Predictive value of serum markers for the development of interstitial lung disease in patients with polymyositis and dermatomyositis: a comparative and prospective study. Intern. Med. J. 45(6), 641-647 (2015).

31. Bandoh S, Fujita J, Ohtsuki Y et al. Sequential changes of KL-6 in sera of patients with interstitial pneumonia associated with polymyositis dermatomyositis. Ann. Rheum. Dis. 59(4), 257-262 (2000).

32. Kubo M, Ihn H, Yamane K et al. Serum KL6 in adult patients with polymyositis and dermatomyositis. Rheumatology (Oxford). 39(6), 632-636 (2000).

33. Pastva AM, Wright JR, Williams KL Immunomodulatory roles of surfactant proteins A and D: implications in lung disease. Proc. Am. Thorac. Soc. 4(3), 252-257 (2007). 
34. Takahashi H, Kuroki Y, Tanaka $\mathrm{H}$ et al. Serum Levels of Surfactant Proteins A and D AreUseful Biomarkers for Interstitial Lung Disease inPatients with Progressive Systemic Sclerosis. Am. J. Respir. Crit. Care. Med. 162(1), 258-263 (2000).

35. Asano $\mathrm{Y}$, Ihn $\mathrm{H}$, Yamane $\mathrm{K}$ et al. Clinical significance of surfactant protein $\mathrm{D}$ as a serum marker for evaluating pulmonary fibrosis in patients with systemic sclerosis. Arthritis. Rheum. 44(6), 1363-1369 (2001)

36. Doyle TJ, Patel AS, Hatabu $\mathrm{H}$ et al. Detection of Rheumatoid Arthritis Interstitial Lung Disease Is Enhanced by Serum Biomarkers. Am. J. Respir. Crit. Care. Med. 191(12), 1403-1412 (2015).

37. Ihn $\mathrm{H}$, Asano $\mathrm{Y}$, Kubo $\mathrm{M}$ et al. Clinical significance of serum surfactant protein D (SPD) in patients with polymyositisdermatomyositis_correlation with interstitial lung disease. Rheumatology (Oxford). 41(11), 1268-1272 (2002).

38. Knoops L, Renauld JC. IL-9 and its receptor: from signal transduction to tumorigenesis. Growth Factors. 22(4), 207-215 (2004).

39. Soroosh P, Doherty TA. Th9 and allergic disease. Immunology. 127(4), 450-458 (2009).

40. Nowak EC, Noelle RJ. Interleukin-9 as a T-helper type 17 cytokine. Immunology. 131(2), 169-173 (2010).

41. Ouyang $H$, Shi $Y$, Liu $Z$ et al. Increased interleukin9 and CD4+IL-9+ T cells in patients with systemic lupus erythematosus. Mol. Med. Rep. 7(3), 10311037 (2013).

42. Yanaba K, Yoshizaki A, Asano Y et al. Serum interleukin 9 levels are increased in patients with systemic sclerosis: association with lower frequency and severity of pulmonary fibrosis. J. Rheumatol. 38(10), 2193-2197 (2011).

43. Jiang $\mathrm{S}$, Wang $\mathrm{Z}$, Ouyang $\mathrm{H}$ et al. Aberrant expression of cytokine interleukin 9 along with interleukin 4 and interferon gamma in connective tissue diseaseassociated interstitial lung disease: association with severity of pulmonary fibrosis. Arch. Med. Sci. 12(1), 101-106 (2016)

44. Corbel M, Theret N, Caulet-Maugendre S et al. Repeated endotoxin exposure induces interstitial fibrosis associated with enhanced gelatinase (MMP2 and MMP-9) activity. Inflamm. Res. 50(3), 129135 (2001).

45. Ohbayashi H. Matrix metalloproteinases in lung diseases. Curr. Protein. Pept. Sci. 3(4), 409-421 (2002).

46. Schwingschackl A, Duszyk M, Brown N et al. Human eosinophils release matrix metalloproteinase-9 on stimulation with TNF-alpha. J. Allergy. Clin. Immunol. 104(5), 983-989(1999).

47. Andersen GN, Nilsson K, Pourazar $\mathrm{J}$ et al. Bronchoalveolar matrix metalloproteinase 9 relates to restrictive lung function impairment in systemic sclerosis. Respir. Med. 101(10), 2199-2206 (2007).
48. Belaaouaj A, Shipley JM, Kobayashi DK et al. Human macrophage metalloelastase. Genomic organization, chromosomal location, gene linkage, and tissue-specific expression. J. Biol. Chem. 270(24), 14568-14575 (1995).

49. Lagente V, Le Quement C, Boichot E. Macrophage metalloelastase (MMP-12) as a target for inflammatory respiratory diseases. Expert. Opin. Ther. Targets. 13(3), 287-295 (2009).

50. Kang HR, Cho SJ, Lee CG et al. Transforming growth factor (TGF)-beta1 stimulates pulmonary fibrosis and inflammation via a Bax-dependent, bid-activated pathway that involves matrix metalloproteinase-12. J. Biol. Chem. 282(10), 77237732 (2007).

51. Matute-Bello G, Wurfel MM, Lee JS et al. Essential role of MMP-12 in Fas-induced lung fibrosis. Am. J. Respir. Cell. Mol. Biol. 37(2), 210-221 (2007).

52. Manetti M, Guiducci S, Romano E et al. Increased serum levels and tissue expression of matrix metalloproteinase-12 in patients with systemic sclerosis: correlation with severity of skin and pulmonary fibrosis and vascular damage. Ann. Rheum. Dis. 71(6), 1064-1072 (2012).

53. Kemp EH, Metcalfe RA, Smith KA et al. Detection and localization of chemokine gene expression in autoimmune thyroid disease. Clin. Endocrinol (Oxf). 59(2), 207-213 (2003).

54. Rotondi M, Rosati A, Buonamano A et al. High Pretransplant Serum Levels of CXCL10/IP-10 Are Related to Increased Risk of Renal Allograft Failure. Am. J. Transplant. 4(9), 1466-1474 (2004).

55. Fujii $\mathrm{H}$, Shimada $\mathrm{Y}$, Hasegawa $\mathrm{M}$ et al. Serum levels of a Th1 chemoattractant IP-10 and Th2 chemoattractants, TARC and MDC, are elevated in patients with systemic sclerosis. J. Dermatol. Sci. 35(1), 43-51 (2004).

56. Tiev KP, Chatenoud L, Kettaneh A et al. Increase of CXCL10 serum level in systemic sclerosis interstitial pneumonia. Rev. Med. Interne. 30(11), 942-946 (2009).

57. Antonelli A, Ferri C, Fallahi P et al. CXCL10 (alpha) and CCL2 (beta) chemokines in systemic sclerosis-a longitudinal study. Rheumatology (Oxford). 47(1), 45-49 (2008).

58. Yoshimura T, Yuhki N, Moore SK et al. Human monocyte chemoattractant protein-1 (MCP-1). Full-length cDNA cloning, expression in mitogenstimulated blood mononuclear leukocytes, and sequence similarity to mouse competence gene JE. FEBS Lett. 244(2), 487-493 (1989).

59. Antoniades HN, Neville-Golden J, Galanopoulos $\mathrm{T}$ et al. Expression of monocyte chemoattractant protein $1 \mathrm{mRNA}$ in human idiopathic pulmonary fibrosis. Proc. Natl. Acad. Sci. U S A. 89(12), 53715375 (1992).

60. Assassi S, Wu M, Tan FK et al. Skin gene expression correlates of severity of interstitial lung disease in systemic sclerosis. Arthritis. Rheum. 65(11), 29172927 (2013). 
61. Amjadi S, Maranian P, Furst DE et al. Course of the modified Rodnan skin thickness score in systemic sclerosis clinical trials: analysis of three large multicenter, double-blind, randomized controlled trials. Arthritis. Rheum. 60(8), 2490-2498 (2009).

62. Assassi S, Sharif R, Lasky RE et al. Predictors of interstitial lung disease in early systemic sclerosis: a prospective longitudinal study of the GENISOS cohort. Arthritis. Res. Ther. 12(5), R166 (2010).

63. Hasegawa M, Sato S, Takehara K. Augmented production of chemokines (monocyte chemotactic protein-1 (MCP-1), macrophage inflammatory protein-1alpha (MIP-1alpha) and MIP-1beta) in patients with systemic sclerosis. Clin. Exp. Immunol. 117(1), 159-165 (1999).

64. Tiev KP, Hua-Huy T, Kettaneh A et al. Serum CC chemokine ligand-18 predicts lung disease worsening in systemic sclerosis. Eur. Respir. J. 38(6), 1355-1360 (2011).

65. Prasse A, Pechkovsky DV, Toews GB et al. CCL18 as an indicator of pulmonary fibrotic activity in idiopathic interstitial pneumonias and systemic sclerosis. Arthritis. Rheum. 56(5), 1685-1693 (2007).
66. Schmidt K, Martinez-Gamboa L, Meier S et al. Bronchoalveoloar lavage fluid cytokines and chemokines as markers and predictors for the outcome of interstitial lung disease in systemic sclerosis patients. Arthritis. Res. Ther. 11(4), R111 (2009).

67. Sakamoto N, Mukae H, Fujii T et al. Differential effects of alpha- and betadefensin on cytokine production by cultured human bronchial epithelial cells. Am. J. Physiol. Lung. Cell. Mol. Physiol. 288(3), L508-513 (2005).

68. Sakamoto N, Kakugawa T, Hara A et al. Association of elevated alpha-defensin levels with interstitial pneumonia in patients with systemic sclerosis. Respir. Res. 16, 148 (2015).

69. Yoshioka S, Mukae H, Ishii $\mathrm{H}$ et al. Alpha-defensin enhances expression of HSP47 and collagen-1 in human lung fibroblasts. Life Sciences. 80(20), 18391845 (2007).

70. Kawasumi H, Gono T, Kawaguchi Y et al. IL-6, IL8, and IL-10 are associated with hyperferritinemia in rapidly progressive interstitial lung disease with polymyositis/dermatomyositis. Biomed. Res. Int. 2014, 815245 (2014) 